\title{
Use of Cement-Augmented Percutaneous Pedicular Screws in the Management of Multifocal Tumoral Spinal Fractures
}

\author{
Mehdi Afathi ${ }^{1}$, Nacer Mansouri ${ }^{2}$, Kaissar Farah ${ }^{2}$, Victor Benichoux ${ }^{3}$, Benjamin Blondel ${ }^{2}$, Stéphane Fuentes ${ }^{2}$ \\ ${ }^{1}$ Neurochirurgie C-Chirurgie du Rachis, Hopital Neurologique et Neurochirurgical Pierre Wertheimer, Hospices Civiles de Lyon, Bron, France \\ ${ }^{2}$ Unité Rachis, Hopital de La Timone, Assistance Publique des Hopitaux de Marseille, Marseille, France \\ ${ }^{3}$ Unité de Génétique et Physiologie de l'Audition, INSERM, Institut Pasteur, Sorbonne Université, Paris, France
}

Study Design: Retrospective case series observational study.

Purpose: Cancer patients are often aged and are further weakened by their illness and treatments. Our goal was to evaluate the efficiency and safety of using minimally invasive techniques to operate on spinal fractures in these patients.

Overview of Literature: Vertebroplasty is now considered to be a safe technique that allows a significant reduction of the pain induced by a spinal tumoral fracture. However, few papers describe the kyphosis reduction that can be achieved by combining percutaneous fixation and anterior vertebral reconstruction.

Methods : We studied 35 patients seen between December 2013 and October 2016 who had at least one pathological spinal fracture and multiple vertebral metastases. The population's mean age was 67 years, and no patients included had preoperative neurological deficits. The patients underwent a minimally invasive surgery consisting of a percutaneous pedicular fixation with cement-enhanced screws and anterior reconstruction comprising kyphoplasty when possible or corpectomy in cases of excessive damage to the vertebral body. Back pain, traumatic local and regional kyphosis, and Beck's Index were collected pre- and postoperatively, and at 3-, 6-, and 12-month follow-ups.

Results : Mean follow-up time was 13.4 months. Significant reductions in back pain $(p<0.001)$ and local $(p<0.001)$ and regional kyphosis $(p=0.006$ ) were found at the 6 -month follow-up (alpha risk level $<0.05)$. Beck's Index was also significantly increased, indicating good restoration of the anterior vertebral height. By the final follow-up, no screws had fallen/pulled out. There were no infectious or neurological complications.

Conclusions: Percutaneous cement-enhanced fixation for pathological fractures has proven a safe and efficient technique in our experience, enabling weak patients to rapidly become ambulatory again without complications. Further follow-up of the patients is necessary to assess the long-term effects of this technique and the continued quality of life of our patients.

Keywords: Metastasis; Minimally invasive surgical procedures; Kyphoplasty; Cementoplasty; Percutaneous spinal fixation

Received Jun 4, 2018; Revised Jul 9, 2018; Accepted Aug 13, 2018

Corresponding author: Mehdi Afathi

Neurochirurgie C-Chirurgie du Rachis, Hopital Neurologique et Neurochirurgical Pierre Wertheimer, Hospices Civiles de Lyon, 59 Boulevard Pinel, 69500 Bron, France

Tel: +33-72357824, Fax: +33-472657262, E-mail: mehdiafathi@gmail.com 


\section{Introduction}

Metastases represent $70 \%$ of spinal tumors and one-third of pathological fractures [1]. Among 100 cancer patients, six will suffer from a vertebral pathological fracture during their lifespan. These patients are fragile, often old, and frequently have multiple spinal metastases. Thus, they should be treated with minimally invasive therapies in order to avoid complications.

Metastatic spinal fractures are most frequently vertebral compression fractures (VCFs) occurring in a bone weakened by the malignant osteolysis. Radiotherapy and, more recently, vertebral augmentation are the main tools used to treat such fractures [2]. The efficiency of these techniques in terms of pain reduction is proven [3-9], although they are insufficient for reducing important kyphotic deformities and treating unstable fractures.

An alternative to these procedures, which overcomes those limitations, consists of percutaneous fixation with cement-augmented screws, followed by a corporeal reconstruction either by balloon kyphoplasty or corpectomy performed with an anterior, minimally invasive approach.

The aim of the study was to evaluate the efficiency and safety of such a treatment in patients with cancer and metastatic spinal fractures.

\section{Materials and Methods}

This retrospective observational study was reviewed by the University of Aix-Marseille Institutional Review Board which concluded that its approval was not necessary, and informed consent were waived. This study is retrospective and includes 35 patients ( 20 women and 15 men) operated on between December 2013 and October 2016 in the neurosurgical department of the University Hospital of Marseille, France. The mean age was 67 years (range, 26-94 years), and all patients had vertebral unstable fractures on pathological vertebrae with a Spinal Instability Neoplastic Score (SINS) [10] greater than or equal to 13 . There were a total of 42 fractures. Eighteen patients suffered from a solid cancer (four breast, four kidney, two melanoma, four thyroid, three lung, and one mediastinal inflammatory myofibroblastic tumor), whereas the remaining $17 \mathrm{pa}-$ tients had hematologic tumors (15 multiple myeloma and two secondary bone lymphomas). All patients suffered from multiple pathological vertebral softening (metastasis or myeloma localization). The subjects underwent pre- operative clinical and radiological assessments and an assessment of back pain using the Visual Analog Scale (VAS). The preoperative radiological checkup included computed tomography (CT) scans with axial, sagittal, and coronal reconstruction, as well as magnetic resonance imaging to detect other vertebral metastases and assess the posterior involvement (necessary to calculate the SINS scores). Surgical treatment was performed on patients with at least one unstable fracture on a metastatic vertebra and for whom spinal cord integrity was not compromised. None of the patients showed neurological signs.

We used only fenestrated pedicular screws to enhance their stability with small volumes of high-viscosity cement, under the working hypothesis that every vertebra in a metastatic spine has a modified and potentially fragile bone structure. The procedure was performed under general anesthesia in the prone position on a radio-transparent spine-operating table, allowing the use of pre- and peroperative frontal and lateral X-ray controls. This 'spine table' induced a spinal lordosis, thus making the fracture reduction easier.

The first stage of the procedure consisted of performing percutaneous osteosynthesis using a Longitude device (Sofamor Danek Medtronic, Memphis, TN, USA) in 33 cases, although a Mantis device (Stryker, Kalamazoo, MI, USA) was used in two cases. Pedicular fenestrated screws were placed under frontal and lateral fluoroscopic control. Long-segment screw fixation (two above and two below) was used in 26 cases; the remaining nine cases included short-segment screw fixation (one level above and one below), bridging only the fractured parts. Once the screws were implanted, cement was injected into each of them under fluoroscopic control. Radio-opaque cement (polymethylmethacrylate [PMMA]) was used in all patients. The third stage of the procedure comprised reducing the

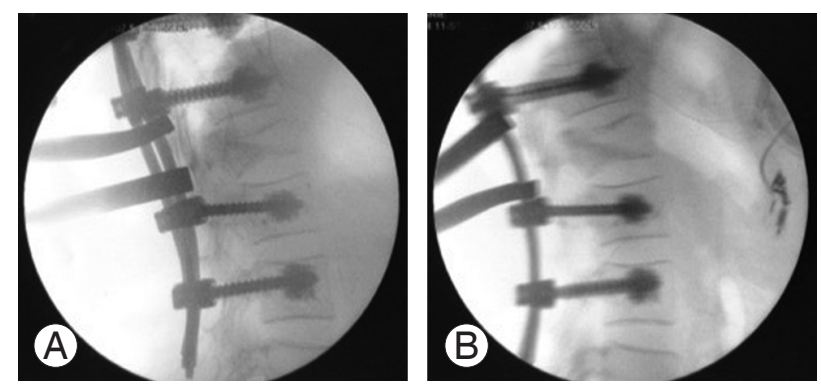

Fig. 1. (A, B) Peroperative fluoroscopic lateral pictures showing a distraction maneuver, performed to reduce the pathological kyphosis. 

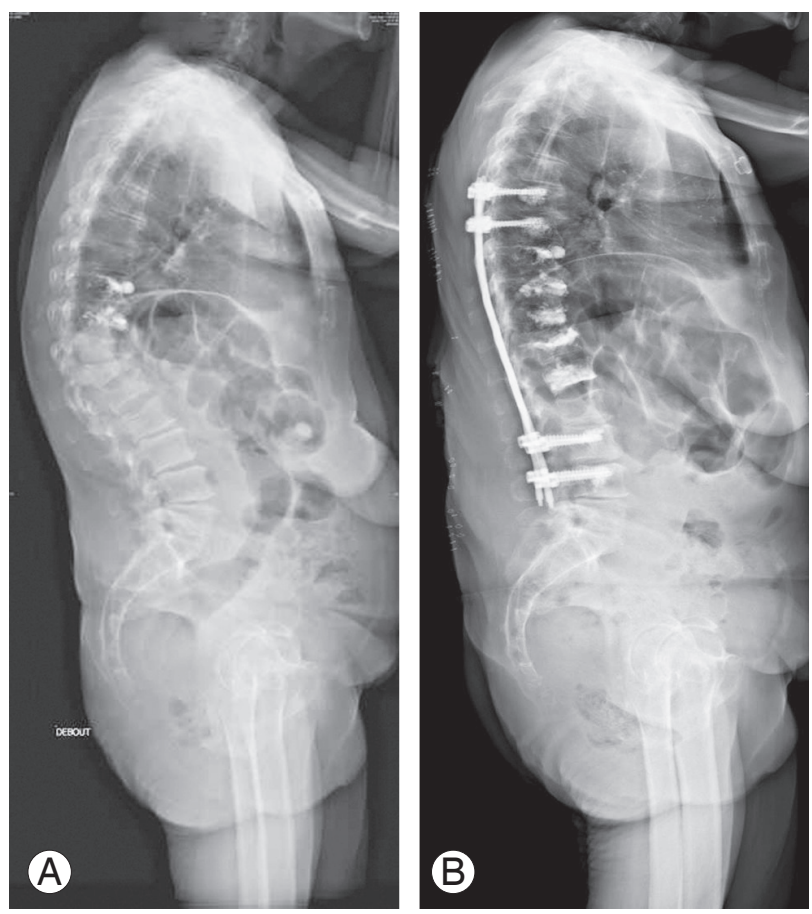

Fig. 2. (A) Lateral full-spine X-ray in a 69-year-old female patient with breast cancer, showing multiple thoracic and lumbar pathological fractures inducing a significant kyphosis. (B) Lateral full-spine X-ray of the same patient taken postoperatively, showing a good reduction of the sagittal deformity.

traumatic kyphosis by locking the screws on the titanium stems, using an in situ lordosing maneuver when necessary (Fig. 1). To avoid having the screws pulled out, this final step was performed at least 15 minutes after the cement injection (allowing it to harden). Fig. 2A and B show pre- and postoperative (respectively) lateral X-ray pictures of a 69-year-old female patient with multimetastatic breast cancer. The thoraco-lumbar kyphosis induced by the pathological fractures was well corrected, allowing the patient to walk with minimal back pain.

In 29 patients (83\%), this procedure was associated with a percutaneous balloon kyphoplasty in the fractured vertebra (Fig. 3), using a Kyphon device (Sofamor Danek Medtronic) to consolidate the reduction and prevent secondary kyphosis. No kyphoplasty was performed when there was too much damage to the vertebral body because of the high risk of cement leakage. In these cases, we performed a complementary corpectomy by the anterior route (two cases, 5\% of the patients). During the postoperative hospital stay, all patients went through follow-up clini$\mathrm{cal}$ and radiological assessments. The clinical assessments comprised a neurological exam and pain evaluation (VAS);
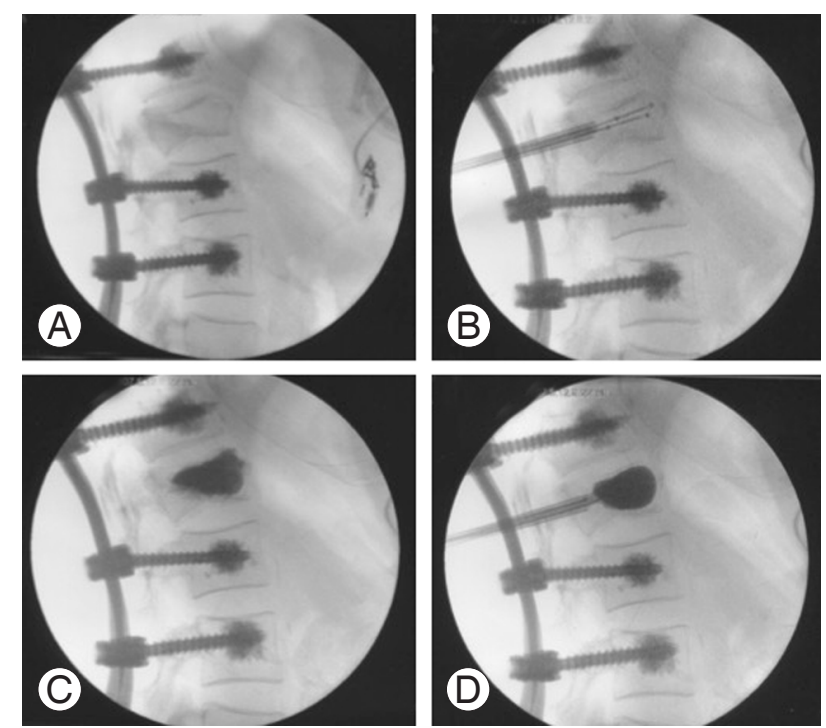

Fig. 3. Peroperative fluoroscopic lateral pictures showing a balloon kyphoplasty. (A) The fractured vertebrae before the kyphoplasty. (B) The introduction of the balloons into the vertebrae. (C) The result after the cement was injected. (D) The balloons inflating.

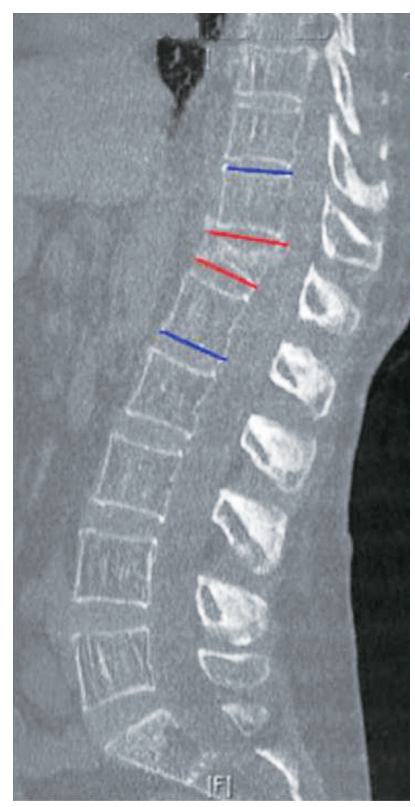

Fig. 4. Lateral computed tomography scan reconstruction of a 65-yearold male patient with myeloma suffering from a D12 pathological fracture. The red lines form the local kyphosis angle, and the blue lines form the regional kyphosis angle.

radiological assessments included a postoperative CT scan with axial, sagittal, and coronal reconstruction to check screw placement and potential cement leakages, as well as to evaluate the angular reduction of the traumatic deformity (kyphosis). The outpatient follow-up consisted of a clinical follow-up, during which CT scans performed at 
3 months, 6 months, and 1 year postoperatively were examined. Only 3 month results were analyzed in this study since not all patients could be followed up for the full year.

Angular reduction of the traumatic deformity was assessed using two values measured on the sagittal CT scan reconstructions (Fig. 4): local kyphosis (LK), defined as the angle between the superior and the inferior endplates of the fractured vertebra; and regional kyphosis (RK), defined as the angle between the superior endplate of the vertebra directly above the fractured one and the inferior endplate of the vertebra directly below the fractured one. Recovery of vertebral height was also measured on sagittal reconstructions using the Beck Index, defined as the ratio of the height of the anterior face of the fractured vertebral body to the height of the posterior face of this vertebra. All these measures were made both pre- and postoperatively.

Results were analyzed statistically using a Shapiro-Wilk test to assess the normality of the distribution. A parametric Student $t$-test and nonparametric Mann-Whitney $U$-test were used to test the significance of the differences between preoperative and 3-month postoperative measurements, with an alpha risk level ( $p$-value) of 0.05 .

\section{Results}

Thirty-five patients with vertebral metastatic fractures (20 men and 15 women) were treated using the described method and included in the study. Mean follow-up was 13.4 months. Mean age was 67 years (range, 26-94 years) at the time of surgery. Lesions were located on vertebrae between T3 and L4, with the most frequent levels of metastatic fractures at T9 and T12 (seven cases each).

No neurological or local infectious complications were found. One patient was found to have a hospital-acquired pulmonary infection after the surgery, two patients were
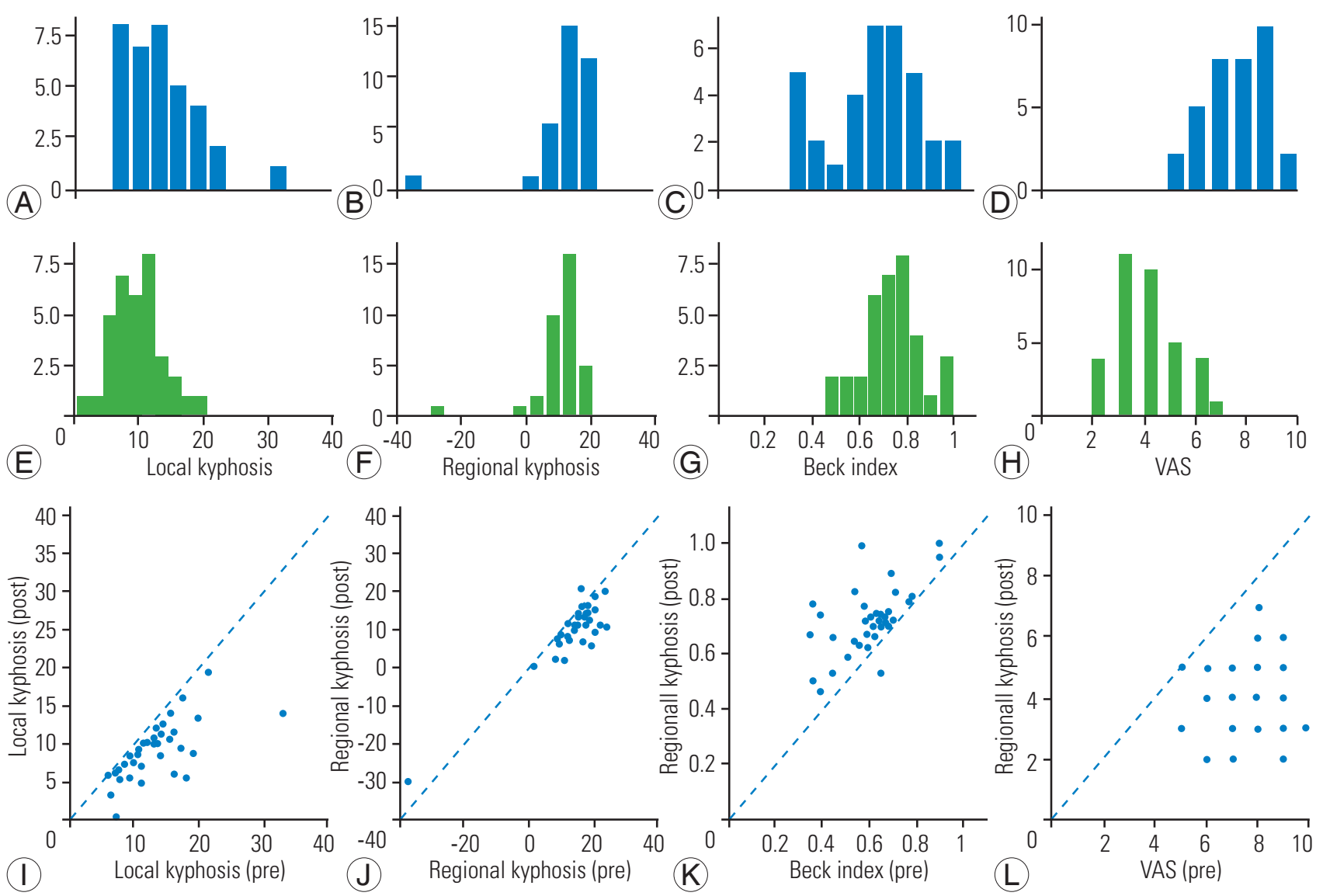

Fig. 5. Pre- vs. postoperative comparisons categorized by measure of interest. (A, E, I) Local kyphosis; (B, F, J) regional kyphosis; (C, G, K) Beck Index; ( $\mathbf{D}, \mathbf{H}, \mathbf{L})$ VAS pain score. (A-H) show the sample histograms for preoperative (A-D, blue) and postoperative (E-H, green) samples. (I-L) show the plots of postoperative vs. preoperative measures for each subject alongside the first diagonal (black dashed line). Points above or below the diagonal indicate decreases or increases of the measure, respectively. VAS, Visual Analog Scale. 
treated for a hospital-acquired urinary infection, and one patient was treated for a pulmonary embolism (due to a deep vein thrombosis). The mean duration of the operations was 79 minutes (range, 65-120 minutes) and that of the hospital stay was 9.5 days (range, $4-30$ days). Screw diameters were $6.5 \mathrm{~mm}$ in lumbar pedicles and 6.5 or 5.5 $\mathrm{mm}$ in thoracic pedicles. We used $40-50 \mathrm{~mm}$ long screws. The mean amount of cement injected was $1.56 \mathrm{~mL}$ (range, 1.2-2.3 mL) per screw. Postoperative CT images showed that the pedicular screws were all correctly placed and that cement leakage occurred in seven cases (20\% of patients), albeit with no clinical consequences (i.e., neurological deficits or pulmonary emboli). Cement leakage occurred through the screws in five cases and through the kyphoplasty in two cases. No pedicle screws had been pulled out as of the last follow-ups. Statistical analyses comparing preoperative data with those of the 3-month postoperative follow-up (LK, RK, and Beck Index) are shown in Fig. 5.

\section{Visual Analog Scale for back pain}

None of the pre- or postoperation samples followed a normal distribution as assessed by a Shapiro-Wilk test ( $W=0.93, p=0.024$ for the 'preoperative' sample; $W=0.92$, $p=0.012$ for the 'postoperative' sample). We tested whether the postoperative VAS pain scores were significantly lower than preoperative scores and found the hypothesis to be true for both a parametric (Student $t$-test, one-sided, paired: $T=13.3, p<0.001)$ and nonparametric (MannWhitney $U$-test: $U=34.00, p<0.001)$ test. The effect was an approximately twofold decrease in VAS (preoperative, 7.71; postoperative, 3.91 ).

\section{Local kyphosis}

Only the preoperation sample followed a normal distribution (preoperative: $W=0.89, p=0.0027$; postoperative: $W=0.98, p=0.721$ ). The postoperative score was significantly lower than the preoperative score in parametric (one-sided $t$-test, paired: $t=5.93, p<0.001)$ and nonparametric $(U=326, p<0.001)$ tests. The average LK measure decreased by $30 \%$, from 13.03 to 9.02 .

\section{Regional kyphosis}

Both pre- and postoperation samples followed a normal distribution $(W=0.59, p<0.001$ and $W=0.71, p<0.001$, re- spectively). Postoperative scores were significantly lower than preoperative scores using both parametric $(t=5.12$, $p<0.001)$ nonparametric $(U=337, p<0.001)$ tests. The average LK measure decreased by approximately $30 \%$, from 13.43 to 9.69 .

\section{Beck Index}

Neither the pre- nor postoperation samples followed a normal distribution ( $W=0.95, p=0.144$ and $W=0.96$, $p=0.252$, respectively). Postoperative Beck indices were significantly higher than preoperative indices using both parametric $(t=-5.97, p<0.001)$ and nonparametric $(U=292.5, p<0.001)$ tests. The Beck Index increased approximately $15 \%$ from pre- to postoperative samples, from 0.6 to 0.71 .

\section{Discussion}

The patient group dealing with metastatic VCF is comparable with that dealing with osteoporotic vertebral fractures. Indeed, both groups are often aged, in poor general health, weakened by comorbidities, and exposed to a high rate of complications. Thus, pathological VCFs need to be managed similarly to osteoporotic VCFs.

Vertebroplasty has proven successful in reducing the pain due to pathological VCF. Many studies report statistically significant pain reductions [11-17]. However, the technique is less useful in reducing kyphotic deformity. Rastogi et al. [18] described vertebroplasty as a painreducing technique that is well tolerated, but only able to prevent kyphosis from worsening. Percutaneous pedicle screw fixation is a minimally invasive technique that can be combined with vertebroplasty, allowing a satisfactory reduction of the traumatic kyphosis with low complication rates and a short operative time. In recent studies, Park et al. [19] and Kim et al. [20] showed that vertebroplasty combined with percutaneous osteosynthesis improves the quality of life (increase of the Eastern Cooperative Oncology Group performance status). Yu et al. [21] measured a significant reduction of traumatic kyphosis in a case series comprising 16 subjects.

Metastatic bone of any type is fragile. Pseudarthrosis and screw-pullout rates are higher in this population, making osteosynthesis more difficult than in other patients. Cement screw enhancement is a good way to contain that risk. Christodoulou et al. [22] demonstrated with 


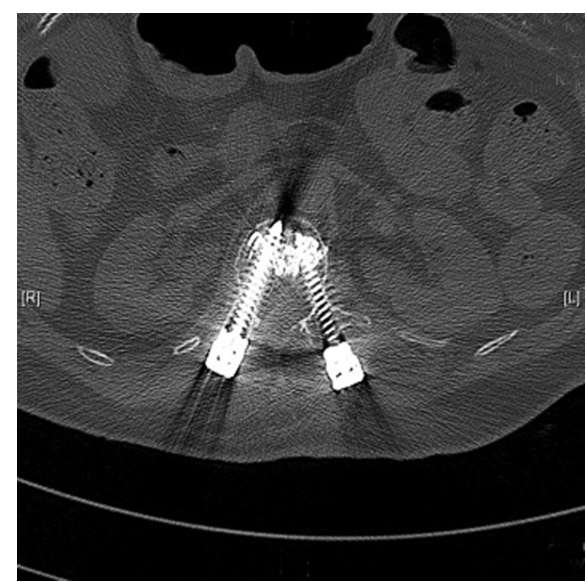

Fig. 6. Axial computed tomography scan reconstruction. To avoid cement leakage, pedicular screws must be long, large, and placed with a convergent trajectory.

their cadaveric study that fenestrated screws have higher axial pullout strength than regular and dual-diameter screws. To our knowledge, only a few studies have been published concerning cement-augmented pedicle screw fixation in pathological VCF. Frankel et al. [23] reported a $20 \%$ pullout rate in their 119 cases, which is disturbingly high. Tan et al. [24] reported a mean operative time of 210 minutes, more than twice our mean time. Our results are instead comparable with those of Pesenti et al. [25], which described cement-enhanced percutaneous screw fixation in osteoporotic VCF. Although their patients did not suffer from the same pathology as ours (osteoporotic versus neoplastic bone), they shared the trait of fragile bone with a high risk of screws pulling out. The authors reported a significant reduction of the VAS and kyphosis, but no significant increase in the Beck Index. The mean amount of cement used was $1.8 \mathrm{~mL}$, and the screws used were 5.5or $6.5-\mathrm{mm}$ diameter fenestrated screws. These similarities may be due to the patients being admitted and treated in the same department; indeed, some were even treated by the same surgeons. Results reported by Moussazadeh et al. [26] are also consistent with ours, demonstrating a significant decrease of the VAS without major complications, and only one case of screw pullout. Therefore, the use of long and large screws in combination with the injection of at least $1 \mathrm{~mL}$ of cement per screw seems to prevent mechanical failures (Fig. 6).

One study recently described results that suggest that cement augmentation of the pedicular screws does not systematically increase their stability. The authors noted that the use of cement augmentation only prevents pulling out in cases of decreased bone mineral density and in cases of filling at least $75 \%$ of the trajectory volume with cement [27].

In our study, two patients underwent a corpectomy because of the extent of destruction of the vertebral body. We acknowledge that this may represent selection bias, but included these two patients (5\% of the population) since their corpectomies were achieved through a quick, minimally invasive lateral retroperitoneal laparotomy without drainage.

Although our cement leakage rate was noticeable, previous studies did not report this measure, so it is hard to evaluate our rate in a broader context. Placing long screws with a convergent trajectory and injecting paste-like PMMA cement are viable techniques to reduce the risk of leakage, especially in the medullar canal.

\section{Conclusions}

Percutaneous cement-augmented screw fixation associated with balloon kyphoplasty or anterior corpectomy and reconstruction is a minimally invasive procedure that allows for quick control of back pain and the reduction of spinal sagittal deformity caused by pathological vertebral fractures. Cement injection in pedicle screws can effectively reduce the number of fixed segments while retaining good screw stability. General complications and infections are rare. Our cement leakage rate was seemingly high (20\%), but was never symptomatic. Patients with cancer have fragile bone and need to be treated quickly, and with the least aggressive therapeutic options, to allow them to undergo their optimal cancer treatments (e.g., radiotherapy and chemotherapy).

\section{Conflict of Interest}

No potential conflict of interest relevant to this article was reported.

\section{References}

1. Curtis JR, Taylor AJ, Matthews RS, et al. "Pathologic" fractures: should these be included in epidemiologic studies of osteoporotic fractures? Osteoporos Int 2009;20:1969-72.

2. Kaloostian PE, Yurter A, Etame AB, Vrionis FD, Sciubba DM, Gokaslan ZL. Palliative strategies for 
the management of primary and metastatic spinal tumors. Cancer Control 2014;21:140-3.

3. Germano IM, Carai A, Pawha P, Blacksburg S, Lo YC, Green S. Clinical outcome of vertebral compression fracture after single fraction spine radiosurgery for spinal metastases. Clin Exp Metastasis 2016;33:143-9.

4. Faivre JC, Py JF, Vogin G, et al. Conformal radiotherapy for vertebral bone metastasis. Cancer Radiother 2016;20:493-9.

5. Stephenson MB, Glaenzer B, Malamis A. Percutaneous minimally invasive techniques in the treatment of spinal metastases. Curr Treat Options Oncol 2016;17:56

6. Bae JW, Gwak HS, Kim S, et al. Percutaneous vertebroplasty for patients with metastatic compression fractures of the thoracolumbar spine: clinical and radiological factors affecting functional outcomes. Spine J 2016;16:355-64.

7. Miller JA, Balagamwala EH, Chao ST, et al. Spine stereotactic radiosurgery for the treatment of multiple myeloma. J Neurosurg Spine 2017;26:282-90.

8. Bhattacharya IS, Hoskin PJ. Stereotactic body radiotherapy for spinal and bone metastases. Clin Oncol (R Coll Radiol) 2015;27:298-306.

9. Health Quality Ontario. Vertebral augmentation involving vertebroplasty or kyphoplasty for cancerrelated vertebral compression fractures: a systematic review. Ont Health Technol Assess Ser 2016;16:1-202.

10. Fisher CG, DiPaola CP, Ryken TC, et al. A novel classification system for spinal instability in neoplastic disease: an evidence-based approach and expert consensus from the Spine Oncology Study Group. Spine (Phila Pa 1976) 2010;35:E1221-9.

11. Montagu A, Speirs A, Baldock J, Corbett J, Gosney M. A review of vertebroplasty for osteoporotic and malignant vertebral compression fractures. Age Ageing 2012;41:450-5.

12. Papanastassiou ID, Aghayev K, Berenson JR, Schmidt $\mathrm{MH}$, Vrionis FD. Is vertebral augmentation the right choice for cancer patients with painful vertebral compression fractures? J Natl Compr Canc Netw 2012;10:715-9.

13. Qian Z, Sun Z, Yang H, Gu Y, Chen K, Wu G. Kyphoplasty for the treatment of malignant vertebral compression fractures caused by metastases. J Clin Neurosci 2011;18:763-7.

14. Tancioni F, Lorenzetti MA, Navarria P, et al. Percuta- neous vertebral augmentation in metastatic disease: state of the art. J Support Oncol 2011;9:4-10.

15. Jensen ME, Kallmes DE. Percutaneous vertebroplasty in the treatment of malignant spine disease. Cancer J 2002;8:194-206.

16. Hirsch JA, Hirsch AE, Jha R, et al. Practical management of malignant compression fractures. J Neurointerv Surg 2010;2:219-20.

17. Masala S, Lunardi P, Fiori R, et al. Vertebroplasty and kyphoplasty in the treatment of malignant vertebral fractures. J Chemother 2004;16 Suppl 5:30-3.

18. Rastogi R, Patel T, Swarm RA. Vertebral augmentation for compression fractures caused by malignant disease. J Natl Compr Canc Netw 2010;8:1095-102.

19. Park HY, Lee SH, Park SJ, Kim ES, Lee CS, Eoh W. Minimally invasive option using percutaneous pedicle screw for instability of metastasis involving thoracolumbar and lumbar spine: a case series in a single center. J Korean Neurosurg Soc 2015;57:100-7.

20. Kim CH, Chung CK, Sohn S, Lee S, Park SB. Less invasive palliative surgery for spinal metastases. J Surg Oncol 2013;108:499-503.

21. Yu WL, Lu JM, Ouyang J, Wei YL, Fang M, Wang XW. Clinical study of open vertebroplasty in treating thoracolumbar metastatic tumor. Zhongguo $\mathrm{Gu}$ Shang 2010;23:739-42.

22. Christodoulou E, Chinthakunta S, Reddy D, et al. Axial pullout strength comparison of different screw designs: fenestrated screw, dual outer diameter screw and standard pedicle screw. Scoliosis 2015;10:15.

23. Frankel BM, Jones T, Wang C. Segmental polymethylmethacrylate-augmented pedicle screw fixation in patients with bone softening caused by osteoporosis and metastatic tumor involvement: a clinical evaluation. Neurosurgery 2007;61:531-7.

24. Tan JW, Shen BH, DU W, Liu JQ, Lu SQ. Bone cement enhanced pedicle screw fixation combined with vertebroplasty for elderly patients with malignant spinal tumors. Chin Med J (Engl) 2013;126:2495-8.

25. Pesenti S, Blondel B, Peltier E, Adetchessi T, Dufour $\mathrm{H}$, Fuentes S. Percutaneous cement-augmented screws fixation in the fractures of the aging spine: is it the solution? Biomed Res Int 2014;2014:610675.

26. Moussazadeh N, Rubin DG, McLaughlin L, Lis E, Bilsky MH, Laufer I. Short-segment percutaneous pedicle screw fixation with cement augmentation for tumor-induced spinal instability. Spine J 
2015;15:1609-17.

27. Fan HT, Zhang RJ, Shen CL, et al. The biomechanical properties of pedicle screw fixation combined With trajectory bone cement augmentation in osteoporotic vertebrae. Clin Spine Surg 2016;29:78-85. 